\title{
New “Bucky-ligands". Potentially Monoanionic Terdentate Diamino Aryl Pincer Ligands Anchored to $\mathbf{C}_{60}$
}

\author{
Michel D. Meijer, Mathias Rump, Robert A. Gossage, Johann H. T. B. Jastrzebski, and Gerard van Koten \\ Debye Institute, Department of Metal-Mediated Synthesis, \\ Utrecht University, Padualaan 8, 3584 CH Utrecht, The Netherlands
}

Received 24 June 1998; accepted 6 July 1998

\begin{abstract}
Two new methanofullerenes have been prepared by the reaction of $\mathrm{C}_{60}$ with diazo substituted, potentially monoanionic, terdentate diamino aryl ligands, yielding a mixture of the open valence $[5,6]$ - and closed valence $[6,6]-$ isomers. Single isomers of the pure [6,6]-methanofullerenes were obtained by heating these mixtures in toluene solution. (1) 1998 Elsevier Science Ltd. All rights reserved.
\end{abstract}

\section{INTRODUCTION}

Fullerenes such as $\mathrm{C}_{60}$ possess a wide variety of chemical and physical properties, which make this class of carbon allotropes not only useful as building blocks in organic chemistry, but also applicable in supramolecular chemistry for the synthesis of molecular devices. The $\mathrm{C}_{60}$ molecule has been shown to be an electron accepting unit ${ }^{1}$. It has also been used in several organic donor-acceptor complexes with for example aniline ${ }^{2}$ or tetrathiafulvalene ${ }^{3}$ or with ligated metal fragments as electron donor groups ${ }^{4-7}$.

Herein we describe the functionalization of $\mathrm{C}_{60}$ with potentially monoanionic terdentate diamino aryl fragments (i.e. 1,3-bis[(dimethylamino)methyl]benzene or "pincer ligand"8) and their use as precursor compounds for aryl metal complexes connected to $\mathrm{C}_{60}$. Attachment of such an electronic rich metal system to $\mathrm{C}_{60}$ may afford an electron push-pull system with novel electronic and photochemical properties. This work may also allow the study of the effect of a para $\mathrm{C}_{60}$ fragment on the catalytic performance of pincer-metal complexes ${ }^{9}$. Compound $\mathbf{A}$ (Figure 1), comprising of $\mathrm{C}_{60}$ bearing a pincer-nickel(II) unit, represents one of our target compounds.

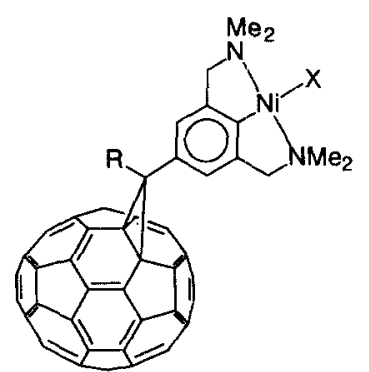

A: $\mathrm{C}_{60}$-pincer-NiX

Figure 1

\section{RESULTS AND DISCUSSION}

There are many possible routes to connect organic groups to fullerenes ${ }^{10}$, but one of the most common methods is a 1,3-dipolar addition of diazo compounds to free $\mathrm{C}_{60}$. This results in the formation of fulleroids and methanofullerenes 10,11 . Using this methodology, we have developed a synthetic route towards methanofullerenes 
containing a pincer ligand. This route starts with the synthesis of hydrazone appended pincer ligands (Scheme 1).

Lithiation (via $\mathrm{Li}$-Br exchange) of 3,5-bis[(dimethylamino)methyl]-1-bromobenzene in $\mathrm{Et}_{2} \mathrm{O}$ at low temperature, followed by a formylation reaction with DMF and a subsequent water quench, produces 1 in excellent yield $(93 \%)$. The aldehyde functionality could be easily converted into a hydrazone by reaction with $\mathrm{N}_{2} \mathrm{H}_{4} \cdot \mathrm{H}_{2} \mathrm{O}$ in EtOH, yielding 2 quantitatively. In a similar way, 4-bromo-3,5-bis[(dimethylamino)methyl]acetophenone ${ }^{12}$ was converted into 3 .
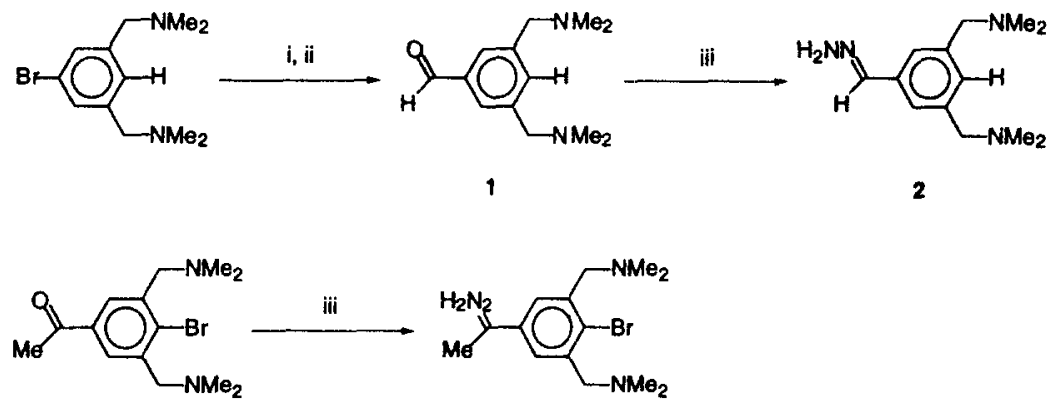

3

SCHEME 1

(i). 2 eq. $t$-BuLi / $\mathrm{Et}_{2} \mathrm{O}$, (ii) DMF, $\mathrm{H}_{2} \mathrm{O}$; (iii) $\mathrm{N}_{2} \mathrm{H}_{4} \cdot \mathrm{H}_{2} \mathrm{O} / \mathrm{EtOH}$

Due to the stability of the hydrazone compounds and the instability of the corresponding diazo-compounds, these were made in situ by oxidation of 2 or 3 with $\mathrm{MnO}_{2}$ in $\mathrm{Et}_{2} \mathrm{O}$. Subsequent addition of the resulting red colored solutions to $C_{60}$ yielded, after column chromatography, a mixture of two products $4 \mathbf{a} / \mathbf{b}$ and $5 \mathbf{a} / \mathbf{b}$, respectively, in a good yield (Scheme 2$)^{13}$.

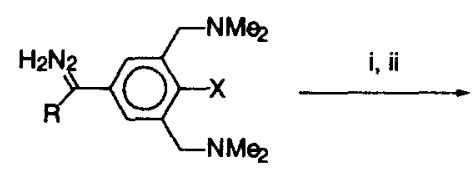

$2 \mathrm{R}=\mathrm{H}, \mathrm{X}=\mathrm{H}$

$3 R=M_{\theta}, X=B r$

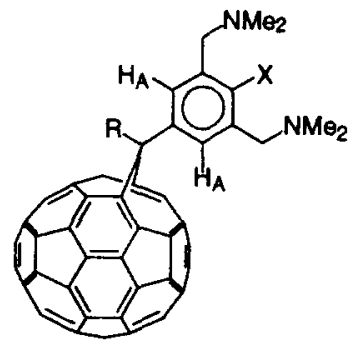

$$
\begin{aligned}
& {[5,6] \text {-isomer }} \\
& \text { 4a } R=H, X=H \\
& 5 a R=M e, X=B r
\end{aligned}
$$

SCHEME 2

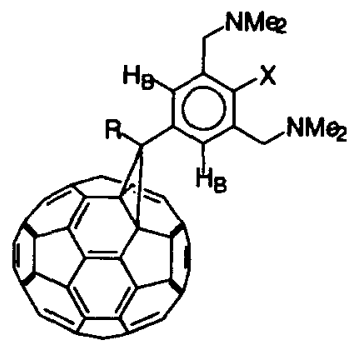

$[6,6]$-isomer

4b $R=H_{1} X=H$

$5 b R=M_{\theta}, X=B r$

(i) $\mathrm{MnO}_{2}, \mathrm{KOH} / \mathrm{EtOH} / \mathrm{Et}_{2} \mathrm{O}$ (ii) $\mathrm{C}_{60} /$ toluene 
$[5,6]$-isomer (4a and $\mathbf{5 a}, 90 \%$ ) with minor amounts of the thermodynamically more stable closed valence $[6,6]$ isomer ( $\mathbf{4 b}$ and $\mathbf{5 b}, \mathbf{1 0 \%}$ ). The compounds $\mathbf{4 a}$ and $\mathbf{4 b}$ exhibit a different chemical shift for the bridgehead proton; while for $\mathbf{5 a}$ and $\mathbf{5 b}$ different signal positions for the aryl protons $\mathrm{H}_{\mathrm{A}}$ and $\mathrm{H}_{\mathrm{B}}$ (Scheme 2) and the bridgehead methyl group are diagnostic NMR resonances $11 \mathrm{~b}$.

To obtain isomerically pure [6,6]-methanofullerenes, toluene solutions of the above reaction mixtures (c.f. scheme 2) were heated at $110^{\circ} \mathrm{C}^{15}$. This yields the pure methanofullerenes $\mathbf{4 b}$ and $\mathbf{5} \mathbf{b}^{16}$, respectively. In the ${ }^{13} \mathrm{C}\left\{{ }^{1} \mathrm{H}\right\}$-NMR spectra ${ }^{15}$, resonances of $\mathrm{sp}^{3}$ hybridized fullerene carbons appeared at $75.6 \mathrm{ppm}(4 \mathrm{~b})$ and 80.6 ppm (5b), confirming the formation of [6.6]-closed valence methanofullerenes ${ }^{10,11}$.

With the preparation of the two methanofullerenes $\mathbf{4 b}$ and $\mathbf{5 b}$, new routes have been opened to the development of novel $\mathrm{C}_{60}$ organometallic compounds that can be used as supported or homogenous catalysts, with the potential for tuning via, for example, modification of the $\mathrm{C}_{60}$ fragment. Preliminary experiments have shown that $\mathbf{5 b}$ can be converted to the corresponding nickel(II) compound via an oxidative addition reaction. Furthermore, lithiation and subsequent transmetallation procedures are being developed to produce $\mathrm{C}_{60}$-pincer metal compounds for which the oxidative addition route is not feasable ${ }^{17}$. The effect of the $\mathrm{C}_{60}$ moiety on these complexes will be reported in due course.

\section{ACKNOWLEDGEMENT}

This work is supported by the Netherlands Foundation of Chemical Research (SON) with financial aid from the Netherlands Organisation for Scientific Research (NWO).

\section{REFERENCES AND NOTES}

1. Allemand, P.-M.; Koch, A.; Wudl, F.; Rubin, Y.; Diederich, F.; Alvarez, M. M.; Whetten, R. L. J. Am. Soc. Chem. 1991, 113, 1050-1051.

2. Williams, R. M.; Zwier, J. M.; Verhoeven, J. W. J. Am. Chem. Soc. 1995, 117, 4093-4099.

3. a) Martin, N.; Sánchez, L.; Seoane, C.; Andreu, R.; Garin, J.; Orduna, J. Tetrahedron Lett. 1996, 37, 5979-5982. b) Martin, N.; Pérez, I.; Sánchez, L.; Seoane, C. J. Org. Chem. 1997, 62, 5690-5695.

4. Ferrocenyl compounds: a) Prato, M.; Maggini, M.; Giacometti, C.; Scorrano, G.; Sandonà, G.; Farnia. G. Tetrahedron 1995, 52, 5221-5234. b) Guldi, D. M.; Maggini, M.; Scorrano, G.; Prato, M. J. Am. Soc. Chem. 1997, 119, 974-980. c) Maggini, M.; Karlsson, A.; Scorrano, G.; Sandonà, G.; Farnia, G.; Prato, M. J. Chem. Soc., Chem. Commmun. 1994, 589-590.

5. Ruthenium systems: a) Sariciftci, N. S.; Wudl, F.; Heeger, A. J.; Maggini, M.; Scorrano, G.; Prato, M.; Bourassa, J.; Ford, P. C. Chem. Phys. Lett. 1995, 247, 510-514. b) Armspach, D.; Constable, E. C.; Diederich, F.; Housecroft, C. E.; Nierengarten, J.-F. Chem. Eur. J. 1998, 4, 723-733. c) Maggini, M.; Donò, A.; Scorrano, G.; Prato, M. J. Chem. Soc., Chem. Commmun. 1995, 845-846.

6. Nickel phthalocyanine adducts: a) Linssen, T. G.; Dürr, K.; Hanack, M.; Hirsch, A. J. Chem. Soc., Chem. Commun. 1995, 103-104. b) Dürr, K.; Fiedler, S.; Linßen, T.; Hirsch, A.; Hannack, M. Chem. Ber./Recueil 1997, 130, 1375-1378. 
7. Zinc-porphyrin systems: a) Ranasinghe, M. G.; Oliver, A. M.; Rothenfluh, D. F.; Salek, A.; Paddon-Row, M. N. Tetrahedron Lett. 1996, 37, 4797-4800. b) Imahori, H.; Hagiwara, K.; Akiyama, T.; Taniguchi, S.; Okada, T.; Sakata, Y. Chem. Lett. 1995, 265-266. c) Imahori, H.; Hagiwara, K.; Aoki, M.; Akiyama, T.; Taniguchi, S.; Okada, T.; Shirakawa, M.; Sakata, Y. J. Am. Chem. Soc. 1996, 118, 11771-11782. d) Kuciauskas, D.; Lin, S.; Seely, G. R.; Moore, A. L.; Moore, T. A.; Gust. D.; Drovetskaya, T.; Reed, C. A., Boyd, P. D. W. J. Phys Chem. 1996, 100, 15926-15932.

8. Rietveld, M. P.; Grove, D. M.; van Koten, G. New J. Chem. 1997, 21, 751-771.

9. a) van de Kuil, L. A.; Grove, D. M.; Gossage, R. A.; Zwikker, J. W.; Jenneskens, L. W.; Drenth, W.; van Koten, G. Organometallics 1997, 16, 4985-4994. b) Granel, C.; Dubois, Ph.; Jérôme, R.; Teyssié, Ph. Macromolecules 1996, 29, 8576-8582.

10. For reviews see: a) Hirsch, A. Synthesis 1995, 895-913. b) Diederich, F.; Thilgen, C. Science 1996, 27I, 317-323.

11. See for example: a) Hummelen, J. C.; Knight, B. W.; Lepeq, F.; Wudl, F.; Yao, J.; Wilkins, C. L. J. Org. Chem. 1995, 60, 532-538. b) Prato, M.; Lucchini, V.; Maggini, M. Stimpfl, E.; Scorrano, G.; Eiermann, M.; Suzuki, T.; Wudi, F. J. Am. Chem. Soc. 1993, 1/5, 8479-8480. c) Prato, M.; Bianco, A.; Maggini, M.; Scorrano, G.; Toniolo, C.; Wudl, F. J. Org. Chem. 1993, 58, 5578-5580.

12. van de Kuil, L. A.; Luitjes, H.; Grove, D. M.; Zwikker, J. W.; van der Linden, J. G. M.; Roelofsen, A. M.; Jenneskens, L. W, Drenth, W.; van Koten, G. Organometallics 1994, 13, 468-477.

13. Yield $4 a / b: 40 \%$ ( $68 \%$ based on reacted $C_{60}$ ), yield $5 a / b: 61 \%\left(91 \%\right.$ based on reacted $C_{60}$ ).

14. Selected ${ }^{1} \mathrm{H}-\mathrm{NMR}$ data (300 $\left.\mathrm{MHz}, \mathrm{CS}_{2} / \mathrm{C}_{6} \mathrm{D}_{6}(3 / 1)\right)$ for $4 \mathbf{a}: \delta=3.93 \mathrm{ppm}$ (bridgehead proton); $4 \mathbf{b}: \delta=$ $5.18 \mathrm{ppm}$ (bridgehead proton); $\mathbf{5 a}: \delta=7.97 \mathrm{ppm}\left(\mathrm{H}_{\mathrm{A}}:\right.$ Scheme 2$), 1.26 \mathrm{ppm}$ (bridgehead methyl); 5b: $\delta=$ $7.99 \mathrm{ppm}\left(\mathrm{H}_{\mathrm{B}}\right.$ : Scheme 2), $2.54 \mathrm{ppm}$ (bridgehead methyl).

15. This type of isomerization is reported to be a zero order reaction, see ref $11 \mathrm{a}$. Therefore, the conversion of the isomer mixtures to $\mathbf{4 b}$ or $\mathbf{5 b}$ was monitored by ${ }^{1} \mathrm{H}-\mathrm{NMR}$ spectroscopy.

16. Data for 4b: ' $\mathrm{H}-\mathrm{NMR}\left(300 \mathrm{MHz}, \mathrm{CS}_{2} / \mathrm{C}_{6} \mathrm{D}_{6}(3 / 1)\right): \delta 7.78$ (s, 2H, aromatic ortho $H$ ); $7.29(\mathrm{~s}, 1 \mathrm{H}$, aromatic $H$ ): $5.17(\mathrm{~s}, 1 \mathrm{H}$, bridge- $\mathrm{CH}) ; 3.39\left(\mathrm{~s}, 4 \mathrm{H}, \mathrm{CH}_{2} \mathrm{~N}\right) ; 2.16\left(\mathrm{~s}, 12 \mathrm{H}, \mathrm{NCH}_{3}\right) ;{ }^{13} \mathrm{C}\left\{{ }^{1} \mathrm{H}\right\}-\mathrm{NMR}(75$ $\mathrm{MHz}_{1} \mathrm{CDCl}_{3}$ ): $\delta 149.75,147.81,145.66,145.56,145.10,145.05,145.02,145.00,144.75,144.60$, $144.48,144.33,144.28,144.05,143.68,143.62,143.01,142.95,142.89,142.83,142.66,142.22$, $142.07,142.02,140.98,140.71,139.45,138.15,137.77,136.40,133.10,130.57,129.81\left(\mathrm{C}_{60}-\mathrm{C}+\mathrm{Ar}-\right.$ C); $75.55\left(\mathrm{C}_{60} \mathrm{sp}^{3}\right) ; 64.11\left(\mathrm{CH}_{2} \mathrm{~N}\right) ; 45.41\left(\mathrm{NCH}_{3}\right) ; 43.47$ (bridgehead-C). Data for 5b: ${ }^{1} \mathrm{H}-\mathrm{NMR}(300$ $\left.\mathrm{MHz}, \mathrm{CS}_{2} / \mathrm{C}_{6} \mathrm{D}_{6}(3 / 1)\right): \delta 7.99(\mathrm{~s}, 2 \mathrm{H}$, aromatic ortho $H) ; 3.54\left(\mathrm{~s}, 4 \mathrm{H}, \mathrm{CH}_{2} \mathrm{~N}\right) ; 2.41\left(\mathrm{~s}, 3 \mathrm{H}, \mathrm{CH}_{3}\right) ; 2.18$ $\left(\mathrm{s}, 12 \mathrm{H}, \mathrm{NCH}_{3}\right) ;{ }^{13} \mathrm{C}\left\{{ }^{1} \mathrm{H}\right\}-\mathrm{NMR}\left(75 \mathrm{MHz}^{\mathrm{CDCl}}{ }_{3}\right): \delta 148.79,147.92,145.93,145.17,145.13,145.11$, $145.07,145.03,144.78,144.73,144.65,144.43,144.34,143.97,143.74,143.70,143.08,142.98$, $142.92,142.14,142.10,141.98,140.96,140.71,138.73,138.26,138.04,137.20,131.86,126.07$ $\left(\mathrm{C}_{60}-\mathrm{C}+\mathrm{Ar}-\mathrm{C}\right) ; 80.63\left(\mathrm{C}_{60^{-}} \mathrm{sp}^{3}\right) ; 63.80\left(\mathrm{CH}_{2} \mathrm{~N}\right) ; 47.19$ (bridgehead-C); $45.54\left(\mathrm{NCH}_{3}\right) ; 22.00\left(\mathrm{CH}_{3}\right)$.

17. Steenwinkel, P.; Gossage, R. A.; van Koten, G. Chem. Eur. J. 1998, 4, 759-762. 